\title{
ALGUNAS CONSIDERACIONES JURÍDICAS PARA ALCANZAR UN CONSENSO EN TORNO A LA GOBERNANZA DE LA LUNA Y LA UTILIZACIÓN DE SUS RECURSOS
}

\author{
SOME LEGAL CONSIDERATIONS TO REACH CONSENSUS \\ REGARDING THE GOVERNANCE OF THE MOON AND THE \\ USE OF ITS RESOURCES
}

Elvira PRADO ALEGRE ${ }^{1}$

Instituto Iberoamericano de Derecho Aeronáutico del Espacio y de la Aviación Comercial, España

\section{Resumen}

Los Estados con mayor capacidad espacial están desarrollando programas para mantener la presencia humana, tanto en la Luna como en su órbita, con el establecimiento de bases lunares y estaciones espaciales. La gobernanza de los retos emergentes, con nuevos actores y nuevos intereses, plantea la necesidad de cuestionarse si éstos deben abordarse dentro o fuera del marco de las Naciones Unidas y si la realidad científica y tecnológica espacial de esta década requiere un mayor desarrollo del derecho espacial y nuevos instrumentos jurídicos para su implementación.

Palabras clave: Derecho espacial, Naciones Unidas, Luna, Recursos espaciales

\begin{abstract}
States with a major space capacity are developing programs to maintain human's presence, both on the Moon and in its orbit, with the establishment of lunar bases and space stations. The governance of emerging challenges, with new actors and new interests, raises the need to question whether these defies should be addressed within or outside the framework of the United Nations system and whether the current space science and technology will require a further development of space law with new legal instruments for its implementation.
\end{abstract}

Keywords: Space Law, United Nations, Moon, Space resources 
SUMARIO: I. Introducción. II. La relevancia de los cinco tratados internacionales para la consolidación del régimen jurídico de la Luna y otros cuerpos celestes. III. Del Acuerdo de la Luna a los Artemis Accords: La historia de un desencuentro. IV. Perspectivas de futuro: Hacia un régimen jurídico efectivo con objetivos compartidos pero diferenciados. V. Conclusiones. VI. Bibliografía.

SUMMARY: I. Introduction. II. The relevance of the five international treaties for the consolidation of the legal regime of the Moon and other celestial bodies. III. From the Moon Agreement to the Artemis Accords: The story of a disagreement. IV. Future prospects: towards an effective legal regime with shared but differentiated objectives. V. Conclusions. VI. Bibliography.

\section{INTRODUCCIÓN}

Coincidiendo con el 60 aniversario del vuelo espacial de Yuri Gagarin, durante la celebración de la Global Space Conference (GLEX) 2021, ${ }^{2}$ las agencias espaciales de la Federación de Rusia y China (ROSCOSMOS y CNSA, China National Space Adminstration) presentaron el proyecto: International Lunar Research Station (ILRS) Guide for partenership, ${ }^{3}$ como continuación al Memorandum de Entendimiento firmado entre ambos organismos en marzo de 2020. El programa científico distingue tres fases de desarrollo que finalizarán con la construcción de infraestructuras en la órbita y en la superficie de la Luna, entre 2026-2035, para su utilización a partir del $2036 .^{4}$ Una alianza que competirá con el Programa Artemisa de la NASA ${ }^{5}$ que dio a conocer sus objetivos en 2017: Liderar la exploración en la Luna con el envío de una misión robótica en 2021, conseguir "llevar la primera mujer y el próximo hombre" al Polo Sur de la Luna hacia 2024, progresar hacia una exploración lunar sostenible a mitad de esta década y establecer una presencia permanente a su final. ${ }^{6}$

Son dos ejemplos de la actividad que están desarrollando las agencias espaciales más importantes del mundo para mantener la presencia humana, tanto en la Luna como en su órbita, con el establecimiento de bases lunares y estaciones espaciales. Los avances científicos y tecnológicos sobre la utilización de los recursos en la Luna durante la última década están transformando el inhóspito satélite en un futuro territorio I+D+I. La utilización de los recursos "in situ", esto es, en suelo extraterrestre (ISRU-In Situ Resource Utilization) para la obtención de aire respirable, agua y energía, ${ }^{7}$ han reactivado la carrera espacial con algunos matices. En el siglo XXI 
la cooperación internacional se ha convertido en la piedra angular de la exploración espacial, por ello, las agencias responsables de estos programas han hecho un llamamiento internacional para que otros Estados se sumen a sus respectivos proyectos. En el caso de la NASA, la cooperación ha trascendido de los ámbitos científicos y tecnológicos al político-jurídico con la elaboración de unos principios, los llamados Artemis Accords, formulados para contribuir a regular la nueva exploración lunar entre los Estados signatarios.

Nos encontramos, por tanto, ante un importante desafío, determinar si el régimen jurídico aplicable en la Luna es suficiente tanto para la regulación de un número más amplio y diverso de actividades espaciales, como para la utilización del suelo lunar y sus recursos, o si, por el contrario, las lagunas existentes requieren la formulación de nuevos principios, normas o tratados que confieran una mayor la seguridad jurídica a los próximos grandes proyectos de exploración lunar, atendiendo a las fases de ejecución de sus programas.

\section{LA RELEVANCIA DE LOS CINCO TRATADOS INTERNACIONALES PARA LA CONSOLIDACIÓN DEL RÉGIMEN JURÍDICO DE LA LUNA Y OTROS CUERPOS CELESTES}

Desde el comienzo de la era espacial la Comisión sobre la Utilización del Espacio Ultraterrestre con Fines Pacíficos de las Naciones Unidas (COPUOS), ha establecido los principios vectores de la exploración espacial a través de tratados, principios y resoluciones de la Asamblea General que hoy conforman el Corpus Iuris Spatialis. ${ }^{8}$ Un régimen jurídico que establece derechos y obligaciones para todos los países que realizan actividades en el espacio ultraterrestre, contribuyendo al fortalecimiento del Estado de derecho. Una exploración que se sustenta esencialmente, como afirma Juan Manuel de Faramiñán Gilbert, ${ }^{9}$ en dos principios claves establecidos en la Carta Magna del espacio de 1967: ${ }^{10}$ La libertad de exploración y utilización del espacio en provecho de todos los países (artículo I) y la no apropiación nacional por reivindicación de soberanía, uso u ocupación (artículo II). Ello no obstante, el derecho espacial contemporáneo vive en constante evolución debido, principalmente, a dos factores: Los avances científicos y tecnológicos y el incremento exponencial de actores públicos y privados en el espacio ultraterrestre, como consecuencia directa de lo anterior. A estos factores, convendría añadir un tercero: el interés creciente en utilizar el territorio lunar y sus órbitas como un espacio para la investigación, el desarrollo y la innovación. No es ésta una cuestión nueva, las delegaciones de la Subcomisión de Asuntos jurídicos de COPUOS llevan más de una década advirtiendo de las posibles 
implicaciones del desarrollo del sector espacial, no solo en la gobernanza de los asuntos espaciales, sino también en la seguridad de dichas actividades.

En su 995ª sesión, celebrada el 31 de mayo de 2021, la Subcomisión de Asuntos Jurídicos de COPUOS volvió a convocar a su Grupo de Trabajo sobre la Situación y Aplicación de los Cinco Tratados de las Naciones Unidas relativos al Espacio Ultraterrestre, bajo la presidencia de Bernhard SchmidtTedd. ${ }^{11}$ Entre los temas examinados tiene especial relevancia la evolución del proyecto de informe elaborado en relación con el "Régimen jurídico del espacio ultraterrestre y la gobernanza global del espacio: perspectivas actuales y futuras". ${ }^{2}$ De su contenido se desprende que la ratificación de los tratados por los Estados permite no solo "participar en un régimen mundial más estable y seguro", sino que además favorece la trasposición de las cláusulas más importantes de los tratados a los instrumentos jurídicos de ámbito nacional, de forma que puedan requerir a las entidades privadas o no estatales el cumplimiento de cuestiones esenciales para la realización de actividades espaciales. ${ }^{13}$ Recordemos, aunque sea brevemente, que en función de lo estipulado en el artículo VI del Tratado del Espacio, los Estados son responsables internacionalmente no solo de las actividades gubernamentales sino también de aquellas realizadas por sus nacionales. ${ }^{14}$

Parece claro, desde la perspectiva del Grupo de Trabajo de la Subcomisión de Asuntos Jurídicos, que la vía que otorga una mayor seguridad jurídica, de cara a un futuro, es la ratificación de los tratados. Conviene señalar que, a la fecha del presente artículo, el Tratado del Espacio Ultraterrestre de 1967 ha sido ratificado por 111 Estados, el Acuerdo de Salvamento de 1968 y el Convenio sobre Responsabilidad de 1972 por 98, respectivamente, el Convenio de Registro de 1975 por 70 Estados, mientras que solo 18 han ratificado el Acuerdo de la Luna de 1979, no siendo ninguno de ellos una potencia espacial. ${ }^{15}$ Esta situación unida a los últimos descubrimientos científicos sobre el suelo lunar, como el agua y las perspectivas de utilización y futura explotación de sus recursos, ${ }^{16}$ han fomentado la promulgación de legislaciones nacionales y acuerdos internacionales, que examinamos a continuación, en detrimento del Acuerdo de la Luna. Por ello, como hemos planteado, una de las cuestiones que debemos analizar es si es pertinente el desarrollo legislativo que demandan no solo algunos Estados con y sin capacidad espacial, sino también representantes de la sociedad civil, para a la utilización de los recursos en la Luna. 


\section{DEL ACUERDO DE LA LUNA A LOS ARTEMIS ACCORDS: LA HISTORIA DE UN DESENCUENTRO}

En la década de los 70, el programa Apolo impulsó la elaboración de un régimen jurídico específico para regular las actividades en la Luna, que concluyó en 1979 tras nueve años de arduas negociaciones en COPUOS. La Asamblea General de las NN.UU. adoptó mediante consenso el Acuerdo que Rige las Actividades de los Estados en la Luna y otros Cuerpos Celestes, convirtiéndose en el único acuerdo que establece en su artículo 11 que "La Luna y los recursos naturales son patrimonio común de la humanidad" y que "Los Estados partes se comprometen a establecer un régimen internacional, incluidos los procedimientos apropiados, que rija la exploración de los recursos naturales de la Luna, cuando esa explotación esté a punto de llegar a ser posible". En 2020, pasados más de cuarenta años, el régimen jurídico de la Luna volvió a ser objeto de estudio, impulsado en esta ocasión por el Proyecto Artemisa de la NASA con la firma de los Artemis Accords, celebrados entre la NASA y ocho Estados signatarios. ${ }^{17}$ Ambos acuerdos nacidos en momentos políticos distintos han sido y son objeto de debate político y jurídico por parte de la comunidad internacional.

Los países que han ratificado el Acuerdo de la Luna como Austria, Australia, México y Bélgica, entre otros, ${ }^{18}$ sostienen que los preceptos del Acuerdo no excluyen la explotación, pública o privada, ni la comercialización de esos recursos, siempre que esa explotación sea compatible con el "patrimonio común de la humanidad". ${ }^{19}$ En el lado opuesto está la posición de los Estados que han legislado sobre la utilización y explotación de los recursos espaciales, o están a favor, como EE.UU. o Luxemburgo, y consideran que esta actividad es compatible con los demás tratados de las Naciones Unidas relativos al espacio ultraterrestre ${ }^{20}$ no siendo necesaria la ratificación del Acuerdo de la Luna. Una controversia doctrinal que no deja de sorprender puesto que el Acuerdo persigue intereses fundamentales para la comunidad internacional en su conjunto. ${ }^{21}$

El heredado desencuentro entre Estados Unidos y la Federación de Rusia, en relación con el Acuerdo de la Luna, se ha mantenido vigente desde la Guerra Fría hasta nuestros días. Si recordamos las posiciones de ambos Estados durante las negociaciones del acuerdo, la antigua Unión Soviética manifestó, desde el principio, su oposición frontal a la sustitución de la expresión en inglés "province of all mankind", 22 empleada en el Tratado del Espacio, por "el patrimonio común de la humanidad". Una discusión que se mantuvo hasta 1979 y que se desbloqueó, como explica Bin Cheng, ${ }^{23}$ no por una aceptación 
del concepto sino por la firma del acuerdo SALT (Second Strategic Arms Limitation Treaty) con EE.UU.

La posición jurídico-política contraria al Acuerdo de la Luna se ha mantenido hasta nuestros días por muchos Estados menos por la propia Federación de Rusia. En su informe presentado en 2014 sobre la situación y aplicación de los cinco tratados, declaró que “(...) La disposición más discutida del Acuerdo sobre la Luna está contenida en el Artículo 11, que establece que la Luna y sus recursos naturales son patrimonio común de la humanidad. Ésta es la única disposición de los tratados de las Naciones Unidas que prevé la posibilidad de explotar los recursos naturales del espacio exterior (...). Al prever la posibilidad y la viabilidad de la explotación de recursos naturales en el espacio exterior, el artículo 11 del Acuerdo sobre la Luna propone una resolución jurídica clara de esta cuestión (...)". ${ }^{24}$ En el mismo informe también manifestó que el Acuerdo no pretendía ser un instrumento jurídico completo ni definitivo, ya que el desarrollo normativo necesario se produciría cuando la explotación de los recursos fuese una realidad. En este mismo sentido, durante las sesiones de 2019, Rusia volvió a considerar la oportunidad de volver a plantear la conveniencia del Acuerdo y una posible ratificación por parte de los Estados. ${ }^{25}$

La posición de Estados Unidos respecto del patrimonio común de la humanidad tuvo un proceso inverso. En mayo de 1972 Herbert Reis, representante de EE.UU. en la Subcomisión de Asuntos Jurídicos, manifestó en relación con los recursos naturales de la Luna y otros cuerpos celestes que, con carácter general, resultaba correcto afirmar que tales recursos forman parte de patrimonio común de toda la humanidad, al igual que los fondos marinos y oceánicos, recordando el apoyo del presidente Nixon a la propuesta. ${ }^{26} \mathrm{Sin}$ embargo, desde la adopción del Acuerdo de la Luna por la Asamblea General de las Naciones Unidas hasta nuestros días, la posición del Senado y de representantes empresariales y asociaciones espaciales siguen considerando que la ratificación del acuerdo limitaría la libertad de explotación de los recursos naturales por parte de las empresas estadounidenses y los derechos de propiedad, en su caso. ${ }^{27}$ No es de extrañar, por tanto, la evolución legislativa en Estados Unidos desde la Commercial Space Launch Act de 1984, reformada en diversas ocasiones, hasta la United States Commercial Space Launch Competitiviness Act de 2015, con un capítulo IV dedicado a la explotación y la utilización de los recursos espaciales y, recientemente, una Executive Order de 6 de abril de 2020 con una expresa mención sobre el Acuerdo "Estados Unidos no es parte del Acuerdo de la Luna. Además, Estados Unidos no considera que el Acuerdo de la Luna sea un instrumento efectivo o necesario 
(...). En consecuencia, el secretario de Estado se opondrá a cualquier intento por parte de cualquier otro Estado u organización internacional de tratar el Acuerdo de la Luna como un reflejo o expresión del derecho internacional consuetudinario". ${ }^{28}$ En este contexto político la NASA anunció el 13 de octubre de 2020 la firma de los Artemis Accords entre la Agencia y varios países socios para el establecimiento de un conjunto práctico de principios que guíen la cooperación entre las naciones para la exploración lunar del siglo $\mathrm{XXI}^{29}$

El objetivo y el alcance de los Artemis Accords, como se expone en los propios acuerdos, se centra en mejorar la seguridad de las operaciones espaciales, reducir incertidumbres y promover el uso sostenible y beneficioso del espacio para toda la humanidad, con la implementación de los principios generales establecidos en el Tratado del Espacio. Con carácter general, los principios enunciados en los Artemis Accords, a través de sus trece secciones, pretenden reforzar el régimen jurídico aplicable a aquellas actividades espaciales civiles, conducidas por las agencias espaciales de cada Estado signatario, en un entorno seguro y transparente que facilite la exploración, la ciencia y las actividades comerciales, habida cuenta del incremento de países y actores del sector privado que llevan a cabo misiones y operaciones en el espacio cislunar. ${ }^{30}$ Muchos de los beneficios que promulgan estos acuerdos coinciden con lo estipulado en el informe del Grupo de Trabajo sobre la aplicación y situación de los Tratados relativos al espacio Ultraterrestre de las Naciones Unidas: ${ }^{31}$ La cooperación internacional para la exploración y la utilización del espacio ultraterrestre con fines pacíficos; la creación de mecanismos de cooperación, la seguridad y la transparencia respecto de los derechos y obligaciones de los Estados, entre otras cuestiones. Los acuerdos reafirman la importancia de los tratados que componen el Corpus Iuris Spatialis a excepción del Acuerdo de la Luna que, como era previsible, no se menciona. De los principios generales, los acuerdos pasan a abordar algunas cuestiones particulares que no recogen los tratados y que analizaremos brevemente, por su especial relevancia.

La Sección 10 aborda la utilización y extracción (no menciona la explotación) de los recursos espaciales, manifestando que ésta deberá realizarse de conformidad con el Tratado del Espacio de 1967, sin que ello implique una apropiación nacional según lo estipulado en el Artículo II del mencionado Tratado ${ }^{32}$. Bajo el epígrafe, en inglés, "Deconfliction of activities" que trata la Sección 11, se establece un sistema para la prevención de conflictos y coordinación de actividades que evite interferencias perjudiciales en las operaciones espaciales, basado en la creación de las denominadas "zonas de 
seguridad". ${ }^{33}$ Otra novedad de los acuerdos es la inclusión de la protección del patrimonio espacial, por su significación histórica de acuerdo con la práctica y los estándares que elaboren los Estados signatarios. ${ }^{34}$

Durante la celebración del 60 periodo de Sesiones de la Subcomisión de Asuntos Jurídicos, se organizó el simposio titulado "Artemis Accords: Towards a Safe and Sustainable Space Exploration" organizado por los Estados signatarios de los acuerdos, con representación de Estados Unidos, Luxemburgo, Japón, Italia y Canadá. ${ }^{35}$ Se expusieron sus ventajas y beneficios como un texto normativo que complementa el Tratado del Espacio para los Estados signatarios, así como la necesidad de adaptar el derecho espacial a la evolución y realidad de las actividades espaciales.

\section{III.1. EL DEBATE JURÍDICO EN TORNO A LA UTILIZACIÓN DE LOS RECURSOS ESPACIALES}

Si bien no hay una posición unánime ni mayoritaria, en cuanto a la conveniencia de ratificar el Acuerdo de la Luna o suscribir los recientes Artemis Accords, sí parece haber mayor consenso respecto de la necesidad de estudiar un posible régimen jurídico para la utilización y la explotación de los recursos espaciales, en general, y de la Luna en particular, que será la opción más realista a corto y medio plazo. ${ }^{36}$ Debemos, en todo caso, distinguir entre la utilización de los recursos "in situ" con fines científicos y su explotación comercial.

La Ley estadounidense de 2015 influenció otras legislaciones como la Ley de Luxemburgo de 2017, ${ }^{37}$ convirtiéndose en el primer país europeo en promulgar una ley específica sobre la apropiación, explotación y utilización de los recursos espaciales. Una cuestión controvertida por el debate político y doctrinal que ha generado sobre la interpretación de los Tratados, que también fue objeto de un análisis profundo en Luxemburgo. El dictamen del Consejo de Estado de Luxemburgo emitido en 2017 puso de manifiesto las incertidumbres que el proyecto de ley generaba tanto en el marco del derecho internacional del espacio, como en relación con el propio derecho nacional. ${ }^{38}$ Entre sus consideraciones señaló que la ley no confería a los operadores la seguridad jurídica deseada, aun siendo el objetivo fundamental de la misma, habida cuenta de las cuestiones jurídicas pendientes respecto de la explotación de los recursos espaciales en el derecho internacional y la ausencia de una posición determinante. A nivel nacional expresó, además, que aun en el supuesto de que Luxemburgo permitiese que los operadores pudiesen apropiarse de 
los recursos no se podría garantizar que otros Estados también aceptasen y reconociesen los derechos de propiedad sobre los mismos. ${ }^{39}$

Como consecuencia de la aparición de legislaciones nacionales, como las señaladas, y los trabajos realizados por Institutos y grupos especializados sobre los recursos espaciales, algunos Estados consideraron necesario centrar el debate sobre la regulación jurídica en el seno de COPUOS y no al margen de las Naciones Unidas. En este sentido las delegaciones de Bélgica y Grecia promovieron en el seno de la Subcomisión de Asuntos Jurídicos, en 2019, la creación de un Grupo de Trabajo sobre posibles modelos de normas jurídicas para las actividades de exploración, explotación y utilización de los recursos ${ }^{40}$ que ha contado con los apoyos de Alemania, Austria, República Checa, Eslovaquia, España, Finlandia, Polonia y Portugal en las sesiones de junio de $2021 .^{41}$ Mención especial, merecen las propuestas presentadas por China y la Federación de Rusia sobre esta cuestión. La propuesta de China ${ }^{42}$ se centra en la creación de un Grupo de Trabajo con un cronograma que finalizaría en 2026 y cuyo eje vector sea el Tratado del Espacio. Los trabajos tendrán en consideración los estudios académicos previos de institutos y centros internacionales, pero insistiendo en que la formulación de nuevas reglas sobre las actividades espaciales debe tener un origen gubernamental de acuerdo con el derecho internacional del espacio y con la práctica establecida por la Comisión. "Por su parte la Federación de Rusia, refuerza la trascendencia de los cinco tratados del espacio, apoyando de esta manera, aunque sin mencionarlo, el Acuerdo de la Luna". Propone, además, que el estudio del Grupo de Trabajo se centre en la identificación de posibles lagunas en el derecho internacional aplicable en relación con la exploración, explotación y utilización de los recursos. Las conclusiones del Grupo podrán contribuir a la elaboración de principios y recomendaciones que puedan sentar las bases para un futuro tratado. ${ }^{43}$

Además de estas propuestas, Luxemburgo y los Países Bajos presentaron un documento de trabajo titulado "Elementos que podrían constituir una base para la elaboración de un marco internacional sobre las actividades relativas a los recursos espaciales". ${ }^{44}$ Ya en 2019, Luxemburgo planteó la necesidad de que el Grupo de Trabajo se guiara por principios políticos que promuevan la inversión pública y privada para la protección tanto los derechos individuales como los intereses colectivos. ${ }^{45}$ Así lo ha vuelto a reiterar durante las sesiones de junio en la Subcomisión, incidiendo en que los debates sobre el marco jurídico que debe regular las actividades relacionadas con los recursos espaciales, tengan en cuenta la labor realizada por otros grupos especializados como Grupo Internacional de Trabajo de La Haya sobre la Gobernanza de los Recursos 
Espaciales $^{46}$ o el European Space Resources Innovation Centre (ESRIC) ${ }^{47}$ con sede en Luxemburgo.

Como hemos podido apreciar las posiciones no solo están divididas en cuanto al contenido sustantivo de ese marco jurídico sino también en relación con el órgano competente para su elaboración. Por un lado, países como China, la Federación de Rusia, Brasil, Alemania, Bélgica, Austria y el Grupo de Estados de Latinoamérica y Caribe (GRULAC), entre otros, defienden el trabajo de COPUOS como foro multilateral, con capacidad para interpretar los tratados y establecer un modelo de gobernanza para la explotación y utilización de los recursos y, por otra parte, hay países que consideran de gran utilidad el trabajo de determinados grupos jurídico-políticos especializados. ${ }^{48}$

\section{PERSPECTIVAS DE FUTURO: HACIA UN RÉGIMEN JURÍDICO EFECTIVO CON OBJETIVOS COMPARTIDOS PERO DIFERENCIADOS}

Las actividades que tienen y tengan lugar, en un futuro, en el espacio ultraterrestre y concretamente en la Luna y sus órbitas, incumben a toda la humanidad por el impacto que pueden tener en nuestro planeta. Su régimen jurídico, por tanto, debería alcanzarse por consenso de todos los Estados con y sin capacidad espacial.

$\mathrm{Si}$ analizamos los objetivos compartidos de forma mayoritaria por los Estados distinguiríamos: La exploración en beneficio de la humanidad, el mantenimiento de la paz, la cooperación internacional, la seguridad de las actividades espaciales, el fortalecimiento del derecho internacional del espacio para la gobernanza de los asuntos espaciales, la sostenibilidad de las actividades y la paulatina participación de la sociedad civil en la búsqueda de soluciones para los problemas y lagunas existentes, entre otros. Son preceptos que aparecen tanto en resoluciones, principios y tratados del espacio como en acuerdos intergubernamentales o en legislaciones nacionales. En un segundo término, derivados de los anteriores, aparecen los objetivos diferenciados de cada Estado, definidos tanto por su capacidad espacial, como por las históricas posiciones jurídico-políticas que han mantenido desde el comienzo de la exploración espacial.

Parece insuficiente para eludir futuros conflictos y mantener la paz en el espacio ultraterrestre que los Estados promulguen legislaciones nacionales sobre cuestiones tan discutidas como la utilización y, sobre todo, explotación de los recursos naturales sin la existencia de un marco jurídico internacional claro sobre esta cuestión. Por otra parte, el incremento del sector privado, 
la participación de la sociedad civil en las actividades espaciales, debido a la constante e imparable transferencia de capacidades y conocimientos del sector público al privado, obligan a contemplarlos como actores necesarios, tal y como aparecen, por ejemplo, en los Artemis Accords. Tampoco podemos obviar a los Estados con menor o nula capacidad espacial, cuya situación implica la necesidad de elaborar un régimen jurídico que también defienda a futuro sus intereses en la Luna, sin paralizar la iniciativa privada que impulsa el devenir del sector espacial. Ello conlleva, a nuestro entender, una adaptación del "patrimonio común de la humanidad" al contexto actual, desde un punto de vista flexible, teniendo en cuenta una serie de variables como las inversiones y riesgos en los que incurren agentes públicos y privados o la experiencia derivada de la explotación de los recursos de los fondos marinos y oceánicos. ${ }^{49}$

La cooperación internacional, la paz y la seguridad, desde un punto de vista jurídico, son objetivos compartidos cuya implementación difiere en función de las distintas posiciones de los Estados respecto del modo en el que deben o pueden alcanzarse. Conviene recordar en este punto el devenir del Código Internacional de Conducta para las Actividades Espaciales, ${ }^{50}$ cuyo proyecto fue presentado durante la Presidencia francesa de la Unión Europea en 2008 y, posteriormente, en las sesiones de la Subcomisión en $2010 .^{51} \mathrm{El}$ código se configuró como un instrumento cuyo objetivo fundamental era reforzar la seguridad y la sostenibilidad de las actividades espaciales. ${ }^{52} \mathrm{Sin}$ embargo, algunas potencias como Rusia y China lo interpretaron como una imposición político-jurídica, aun no siendo vinculante, al haberse elaborado por los Estados de la Unión Europea fuera del marco de la Comisión sobre la Utilización del Espacio Ultraterrestre. ${ }^{53}$ Por su parte, Estados Unidos anunció en 2012 que no suscribiría el proyecto de Código. ${ }^{54}$

Encontrar soluciones pragmáticas que disipen las lagunas del actual régimen jurídico, es otro objetivo compartido que difiere en cuanto al instrumento jurídico final, pudiendo haber cierto consenso en alcanzar primero unos principios o reglas comunes. Considerando la trascendencia, como hemos mencionado, de las actividades espaciales en la Luna y su implicación con otros retos que se debaten en la Subcomisión de Asuntos Jurídicos de COPUOS, como la sostenibilidad del medio ambiente espacial, el tráfico espacial o la reducción de los desechos espaciales, parece lógico pensar que las Naciones Unidas sea el foro intergubernamental idóneo para la negociación de un futuro marco jurídico en beneficio de la humanidad. ${ }^{55}$ De otra parte, habida cuenta del estado actual del desarrollo científico y tecnológico, no solo para la utilización y explotación de los recursos espaciales en la Luna, sino también para el establecimiento de una presencia humana permanente en el satélite, 
parece viable que continúe el estudio de su régimen jurídico dentro del marco de la Comisión y su Subcomisión de Asuntos Jurídicos. En este sentido, sería conveniente que los debates sobre esta cuestión no paralicen la evolución de los trabajos y, para ello, debemos contemplar dos factores decisivos: la voluntad política de los Estados con capacidad espacial para llegar a un consenso sobre las cuestiones consideradas claves en la exploración espacial y la dinámica de la propia Comisión.

Juan Manuel de Faramiñán Gilbert ${ }^{56}$ plantea la conveniencia de retomar la idea en torno a la creación de una Organización Internacional del Espacio que surgió en 1968 durante la Primera Conferencia de las Naciones Unidas para la exploración y utilización pacífica del espacio ultraterrestre (UNISPCE-I). Hasta que no contemos con un organismo como el propuesto, consideramos positiva y necesaria la contribución en COPUOS de la sociedad civil, el sector privado, las organizaciones no gubernamentales y el mundo académico, ${ }^{57}$ habida cuenta del incremento progresivo de su participación en la resolución de los desafíos que plantean las actividades espaciales. Su convivencia con el trabajo de las delegaciones de la Subcomisión parece necesaria, de forma que la realidad no solo jurídica y tecnológica sino también la económica y social, tengan cabida dentro de los desafíos que plantean las actividades espaciales en la Luna. De otra parte, no podemos olvidar el factor tiempo. Si bien los desafíos no son inmediatos y los objetivos trazados por las agencias espaciales no se alcanzarán hasta finales de esta década o mediados de la siguiente, el estudio de las cuestiones aquí analizadas no puede prolongarse ad infinitum en la Subcomisión de Asuntos Jurídicos, como en el caso de la consabida cuestión sobre la definición y delimitación del espacio ultraterrestre. Un tema que permanece en el programa de la Subcomisión desde 1967 y, mientras se sigue debatiendo, ya ha sido testigo en 2021 de los primeros vuelos espaciales suborbitales tripulados con fines turísticos por Richard Brandson (Virgin Galactic) $)^{58}$ y Jeff Bezos (Blue Origin). ${ }^{59}$

\section{CONCLUSIONES}

Alcanzar un consenso sobre el régimen jurídico aplicable para la exploración de la Luna y la utilización de sus recursos, implica debatir sobre las cuestiones objeto de controversia, determinar cuál podrá ser el instrumento jurídico adecuado y el órgano competente y, finalmente, establecer unos plazos que lo hagan posible.

En relación con las cuestiones sustantivas sobre las que se debe de alcanzar un consenso, consideramos oportuno analizar objetivos compartidos pero 
diferenciados, que tengan en cuenta las características y necesidades de los Estados, según su mayor o menor capacidad espacial, con variables como la seguridad jurídica que demanda el sector privado o la adaptación del concepto sobre "el patrimonio común de la humanidad" a la realidad científica y tecnológica de los programas espaciales y a los intereses de la sociedad civil.

Respecto del instrumento jurídico más apropiado, resulta claro, como confirman los informes del Grupo de Trabajo sobre la Situación y Aplicación de los Cinco Tratados de las Naciones Unidas relativos al Espacio y, en concreto, sobre la gobernanza y el régimen jurídico del espacio ultraterrestre, que la ratificación de los tratados es la vía que otorga una mayor seguridad jurídica. Para el mantenimiento de la paz en el espacio ultraterrestre parece insuficiente la formulación de acuerdos generales, por parte de algunos Estados, o la promulgación de legislaciones nacionales. Las implicaciones jurídico-políticas de las futuras actividades en la Luna son extremadamente complejas y requerirán de un detallado corpus normativo. Ahora bien, para lograr un consenso dicho instrumento podrá contener, en una primera fase, unos principios comunes, vinculantes o no, que podrían evolucionar hacia un tratado.

Para determinar cuál es el órgano competente es necesario tener presente que las actividades que tienen lugar en el espacio ultraterrestre y, concretamente, en la Luna y sus órbitas, incumben a toda la humanidad por su impacto planetario. Su régimen jurídico, por tanto, debería alcanzarse por consenso de todos los Estados con y sin capacidad espacial. En este sentido, la Comisión sobre la Utilización del Espacio Ultraterrestre con Fines Pacíficos parece el órgano intergubernamental preferente para lograr este fin. No obstante, la Comisión debe dar cabida a los trabajos realizados por Institutos y grupos especializados, con una mayor contribución de la sociedad civil, que refleje el incremento de su participación en las actividades espaciales.

Por último, no podemos olvidar la importancia del tiempo invertido en la búsqueda de un consenso, tanto en relación con las sesiones y trabajos que tienen lugar en las dos Subcomisiones de COPUOS, como con la previsibilidad de los avances tecnológicos y los programas actuales de las agencias espaciales. Hasta finales de la presente década o principios de la siguiente es probable que no se alcancen muchos de los objetivos pautados, por lo que aún hay una oportunidad, tal vez la última, para llegar a un consenso desde la multilateralidad. 


\section{BIBLIOGRAFÍA}

CHENG, B. (1997), Studies in international space Law, Clarendon Press Oxford.

CLARK, S. (9 de febrero de 2020), "What if we mined the Moon?", Science Focus, https://www.sciencefocus.com/space/what-if-we-mined-the -moon/ (consulta: 2.05.2021).

CONSEIL D'ÉTAT DE LUXEMBOURG. Avis 51.987 du 7 avril 2017. Projet de loi sur l'exploration et l'utilisation des ressources de l'espace, htt ps://conseil-etat.public.lu/fr/avis/2017/04/07042017/51987.html, (consulta: 20.04.2021).

COMMITTEE ON COMMERCE, SCIENCE, AND TRANSPORTATION. UNITED STATES SENATE (1980), Agreement Governing the Activities of States on the Moon and other Celestial Bodies. Prepared at the request of Hon. Howard W. Cannon, President.

FARAMIÑAN GILBERT, J. M. (2009), "Las actividades en la zona internacional de los fondos marinos y en la luna y otros cuerpos celestes (análisis comparativo del aprovechamiento de los recursos)", en FARAMIÑÁN GILBERT, J. M. y MUÑOZ RODRÍGUEZ, C., Convergencias y divergencias de los regímenes jurídicos del espacio ultraterrestre y de determinados espacios marinos, Universidad de Jaén, Jaén, p. 1-22.

FARAMIÑAN GILBERT, J. M. de (2020), Las controvertidas cuestiones sobre la minería espacial. Lagunas jurídicas en la regulación del espacio ultraterrestre, Editorial Kinnamon.

FROEHLICH, A. y SEFFINGA, V. (Eds.) (2018), "National Space Legislation", Studies in Space Policy, Springer International Publishing AG, 15, https://doi.org/10.1007/978-3-319-70431-9_3

HOBE, S. (2010), “The Moon Agreement: Let's Use the Chance!”, $Z L W, \mathrm{n}^{\circ}$ 3 , pp. 372-381.

HOBE, S. (2013), "The 1979 Agreement Governing the Activities of States on the Moon and Other Celestial Bodies. Future Perspectives", en HOBE, S.; SCHMIDT-TEDD, B. y KAI-UWE, S. (Eds.), Cologne Commentary on Space Law Volume II, Carl Heymanns Verlag, Colonia, pp. 423-426.

JOHNSON, C. (2014), "Draft International Code of Conduct for Outer Space Activities Fact Sheet". Secure World Foundation, https://swfound.org/medi a/166384/swf_draft_international_code_of_conduct_for_outer_space_acti vities_fact_sheet_february_2014.pdf, (consulta: 10.06.2021). 
MARCHISIO, S. (2010), "The draft code of conduct for outer space activities", United Nations Workshop on Space Law Activities of States in Outer Space in Light of New Developments: Meeting International Responsibilities and Establishing National Legal and Policy Frameworks, 16-19 November, Bangkok, Thailand, https://www.unoosa.org/pdf/pres/2010/SLW2010/02-1 0b.pdf, (consulta: 1.05.2021).

NASA (septiembre de 2020), NASA's Lunar Exploration Program Overview National Aeronautics and Space Administration PLAN, https://www.nas a.gov/sites/default/files/atoms/files/artemis_plan-20200921.pdf, (consulta: 1.05.2021).

NASA (13 de octubre de 2020), The Artemis Accords principles for cooperation in the civil exploration and use of the Moon, Mars, comets, and asteroids for peaceful purposes,

PRADO ALEGRE, E. (2014), "Sobre el Acuerdo de la Luna y el Patrimonio Cultural del Espacio", XLI Jornadas Iberoamericanas de Derecho Aeronáutico y del Espacio y de la Aviación Comercial. Instituto Iberoamericano de Derecho Aeronáutico del Espacio y de la Aviación Comercial.

PRADO ALEGRE, E. (2018), Los Estados de la Unión Europea frente a los Grandes Retos del Derecho Internacional del Espacio, Instituto Iberoamericano de Derecho Aeronáutico del Espacio y de la Aviación Comercial, Madrid.

PRADO ALEGRE, E. (2020), “Sobre la exploración, explotación y utilización de los recursos de la Luna", Tiempo de Paz, $\mathrm{n}^{\circ} 136$.

ROSCOSMOS, CNSA (junio de 2021), International Lunar Research Station (ILRS) Guide for partnership, https://www.roscosmos.ru/media/files/mnls. pdf, (consulta: 1.05.2021).

SHEETZ, M. (20 de julio de 2021), “Jeff Bezos reaches space on Blue Origin's first crewed launch". CNBC, https://www.cnbc.com/2021/07/20/jeff -bezos-reaches-space-on-blue-origins-first-crewed-launch.html, (consulta: 20.07.2021).

WATTLES, J. (12 de julio de 2021), "Virgin Galactic founder Richard Branson successfully rockets to outer space", $C N N$ Business, https://edition.cnn.com/2021/07/11/tech/richard-branson-virgingalactic-space-flight-scn/index.html, (consulta: 12.07.2021).

WILLIAMS, M. (2010), "The Moon Agreement in Current Scenarios. Amendments to Multilateral Treaties. How the Suggestion Amendments be Made", Proceedings of the international Institute of Space Law 2010. 


\section{3rd Colloquium on the Law of Outer Space, Praga, República Checa (27 septiembre- 1 octubre).}

1 Elvira Prado Alegre. Doctora en Derecho Internacional Público. Instituto Iberoamericano de Derecho Aeronáutico del Espacio y de la Aviación Comercial. Organismo observador de la Comisión para la Utilización del Espacio Ultraterrestre con Fines Pacíficos.

2 La Conferencia, coorganizada por la Federación Astronáutica Internacional (IAF) y ROSCOSMOS tuvo lugar en San Petersburgo del 14 al 18 de junio.

3 ROSCOSMOS, CNSA (junio de 2021), International Lunar Research Station (ILRS) Guide for partnership, https://www.roscosmos.ru/media/files/mnls.pdf, (consulta: 1.05.2021).

$4 \quad$ Ibid.

5 NASA (septiembre de 2020), NASA's Lunar Exploration Program Overview National Aeronautics and Space Administration PLAN, https://www.nasa.gov/sites/default/files/atoms/f iles/artemis_plan-20200921.pdf, (consulta: 1.05.2021).

6 Ibid

7 PRADO ALEGRE, E. (2020), "Sobre la exploración, explotación y utilización de los recursos de la Luna", Tiempo de Paz, no 136, pp. 9 y 10.

8 Los cinco tratados de las Naciones Unidas relativos al espacio ultraterrestre son: el Tratado sobre los Principios que Deben Regir las Actividades de los Estados en la Exploración y Utilización del Espacio Ultraterrestre, incluso la Luna y otros Cuerpos Celestes, 1967 (Tratado sobre el Espacio Ultraterrestre); el Acuerdo sobre el Salvamento y la Devolución de Astronautas y la Restitución de Objetos Lanzados al Espacio Ultraterrestre, 1968 (Acuerdo sobre Salvamento); el Convenio sobre la Responsabilidad Internacional por Daños Causados por Objetos Espaciales, 1972 (Convenio sobre la Responsabilidad); el Convenio sobre el Registro de Objetos Lanzados al Espacio Ultraterrestre, 1975 (Convenio sobre el Registro) y el Acuerdo que Rige las Actividades de los Estados en la Luna y otros Cuerpos Celestes, 1979 (Acuerdo sobre la Luna). NACIONES UNIDAS. Oficina de Asuntos del Espacio Ultraterrestre (UNOOSA). Derecho Internacional del Espacio: Instrumentos de las Naciones Unidas, https://www.unoosa.org/oosa /en/ourwork/spacelaw/treaties.html (consulta: 5.04.2021).

9 FARAMIÑAN GILBERT, J. M. de (2020), Las controvertidas cuestiones sobre la minería espacial. Lagunas jurídicas en la regulación del espacio ultraterrestre, Editorial Kinnamon, p. 16.

10 El Tratado sobre los Principios que Deben Regir las Actividades de los Estados en la Exploración y Utilización del Espacio Ultraterrestre incluso la Luna y otros Cuerpos Celestes (1967).

11 UN Doc. A/AC.105/C.2/2021/TRE/L.1. Proyecto de informe Anexo I Informe de la Presidencia del Grupo de Trabajo sobre la Situación y Aplicación de los Cinco Tratados de las Naciones Unidas relativos al Espacio Ultraterrestre. Subcomisión de Asuntos Jurídicos $60^{\circ}$ período de sesiones Viena, 31 de mayo a 11 de junio de 2021.

12 UN Doc. A/AC.105/C.2/L.313 Proyecto revisado de documento de orientación relativo a la prioridad temática 2 de UNISPACE +50 : Régimen jurídico del espacio ultraterrestre y la gobernanza global del espacio: perspectivas actuales y futuras. Documento de trabajo presentado por la Presidencia del Grupo de Trabajo sobre la Situación y Aplicación de los Cinco Tratados de las Naciones Unidas relativos al Espacio Ultraterrestre. Subcomisión de Asuntos Jurídicos $59^{\circ}$ período de sesiones Viena, 23 de marzo a 3 de abril de 2020.

13 Nos referimos a cuestiones tan importantes como la expedición de licencias, el registro de los objetos espaciales, los seguros e indemnizaciones, en su caso, así como la responsabilidad y la seguridad de las operaciones.

14 Tratado del Espacio Ultraterrestre. Artículo VI: "Los Estados Partes en el Tratado serán responsables internacionalmente de las actividades nacionales que realicen en el espacio ultraterrestre, incluso la Luna y otros cuerpos celestes, los organismos gubernamentales o 
las entidades no gubernamentales, y deberán asegurar que dichas actividades se efectúen en conformidad con las disposiciones del presente Tratado. Las actividades de las entidades no gubernamentales en el espacio ultraterrestre, incluso la Luna y otros cuerpos celestes, deberán ser autorizadas y fiscalizadas constantemente por el pertinente Estado Parte en el Tratado (...)".

15 UN Doc. A/AC.105/C.2/2021/CRP.10. Status of International Agreements relating to activities in outer space as at 1 January 2021.

16 CLARK, S. (9 de febrero de 2020), "What if we mined the Moon?", Science Focus, https://ww w.sciencefocus.com/space/what-if-we-mined-the-moon/ (consulta: 2.05.2021).

17 Hasta junio de 2021, 12 países han adoptado los Acuerdos de Artemis: Australia, Brasil, Canadá, Italia, Japón, Luxemburgo, Nueva Zelanda, República de Corea, Ucrania, Emiratos Árabes Unidos, Reino Unido y Estados Unidos. NASA. The Artemis Accords, https://www.nasa.gov /specials/artemis-accords/index.html.

18 UN Doc. A/AC.105/C.2/2021/CRP.10 (2021). Status of International Agreements relating to activities in outer space as at 1 January 2021. El Acuerdo de la Luna ha sido ratificado por Australia, Armenia, Bélgica, Kazajistán, Kuwait, Líbano, México, Pakistán, Arabia Saudita, Turquía, Venezuela, Chile, Filipinas, Austria, Países Bajos, Marruecos, Uruguay y Perú. UN Doc. A/AC.105/C.2/L.272 (2018). Joint statement on the benefits of adherence to the Agreement Governing the Activities of States on the Moon and Other Celestial Bodies by States parties to the Agreement.

20 Audio de las sesiones de la Subcomisión de Asuntos Jurídicos sobre su $58^{\circ}$ período de sesiones.05.04.2019, https://conferences.unite.un.org/carbonweb/public/oosa/speakerslog/de8 6144b-3fa4-4ac3-a75c-17979298b18f, (consulta: 1.05.2021).

21 FARAMIÑÁN GILBERT, (2020), p. 27; HOBE, S. (2010), “The Moon Agreement: Let's Use the Chance!", ZLW, no 3, pp. 372-381; HOBE, S. (2013), “The 1979 Agreement Governing the Activities of States on the Moon and Other Celestial Bodies. Future Perspectives", en HOBE, S.; SCHMIDT-TEDD, B. y KAI-UWE, S. (Eds.), Cologne Commentary on Space Law Volume II, Carl Heymanns Verlag, Colonia, pp. 423-426.

22 La expresión “(...) Shall be the province of all mankind” que aparece en el artículo I del Tratado del Espacio, figura en la versión española como “(...) Incumben a toda la humanidad” y en la versión francesa como “(...) l'apanage de l'humanité tout entière”. CHENG, B. (1997), Studies in international space Law, Clarendon Press Oxford, pp. 362 y ss.

24 UN Doc. A/AC.105/C.2/2014/CRP.18.2014: “(...) this is the only provision in the United Nations space treaties that provides for the possibility of exploiting the natural resources of outer space. According to international law, such exploitation is not prohibited but must be considered in the light of compliance with the principles applicable to outer space, in particular article II of the Outer Space Treaty. By providing for the possibility and practicability of exploiting natural resources in outer space, article 11 of the Moon Agreement proposes a clear legal resolution of this question under the conditions of compliance set forth in article II of the Outer Space Treaty and other principles of space law".

25 Audio de las sesiones de la Subcomisión de Asuntos Jurídicos sobre su $58^{\circ}$ período de sesiones, del punto 14 del programa. 05.04.2019, https://conferences.unite.un.org/carbonweb /public/oosa/speakerslog/03806dc1-af87-4384-9be8-7e89f2c10840 (min. 11:13:08) y sesiones del 8.04.2019, https://conferences.unite.un.org/carbonweb/public/oosa/speakerslog/5002f7d0a19f-4744-9790-2b9cad1e6999, (consulta: 1.06.2021).

26 COMMITTEE ON COMMERCE, SCIENCE, AND TRANSPORTATION. UNITED STATES SENATE (1980), Agreement Governing the Activities of States on the Moon and other Celestial Bodies. Prepared at the request of Hon. Howard W. Cannon, President, p.14.

27 PRADO ALEGRE, E. (2014), "Sobre el Acuerdo de la Luna y el Patrimonio Cultural del Espacio", XLI Jornadas Iberoamericanas de Derecho Aeronáutico y del Espacio y de la Aviación Comercial. Instituto Iberoamericano de Derecho Aeronáutico del Espacio y de la Aviación Comercial, p. 208.

28 FARAMIÑÁN GILBERT, (2020), pp. 39 y ss.

29 NASA (13 de octubre de 2020,). The Artemis Accords principles for cooperation in the civil exploration and use of the Moon, Mars, comets, and asteroids for peaceful purposes, https://www.nasa.gov/specials/artemis-accords/img/Artemis-Accords-signed-13Oct 2020.pdf, (consulta: 01.05.2021). 
30

31 UN Doc. A/AC.105/C.2/L.310, 2019. Documento de trabajo presentado por la Presidencia del Grupo de Trabajo sobre la Situación y Aplicación de los Cinco Tratados de las Naciones Unidas relativos al Espacio Ultraterrestre. Punto II.A: Consideraciones generales sobre los beneficios, derechos y obligaciones de pasar a ser Estado parte en los tratados relativos al espacio ultraterrestre.

32 The Artemis Accords. Section 10.2.: "The Signatories emphasize that the extraction and utilization of space resources, including any recovery from the surface or subsurface of the Moon, Mars, comets, or asteroids, should be executed in a manner that complies with the Outer Space Treaty and in support of safe and sustainable space activities. The Signatories affirm that the extraction of space resources does not inherently constitute national appropriation under Article II of the Outer Space Treaty, and that contracts and other legal instruments relating to space resources should be consistent with that Treaty".

33 Section 11.7: (...) "A safety zone should be the area in which nominal operations of a relevant activity or an anomalous event could reasonably cause harmful interference (...)". "(c) The nature and existence of safety zones is expected to change over time reflecting the status of the relevant operation. If the nature of an operation changes, the operating Signatory should alter the size and scope of the corresponding safety zone as appropriate. Safety zones will ultimately be temporary, ending when the relevant operation ceases (...)".

34 The Artemis Accords. Section 9. Preserving Outer Space Heritage.

35 UNOOSA. Artemis Accords: Safe and Sustainable Space Exploration. Online event (02.06.2021), https://www.unoosa.org/documents/pdf/copuos/lsc/2021/Artemis_Accords_Saf e_and_Sustainable_Space_Exploration_1.pdf, (consulta: 3.06.2021).

36 WILliaMS, M. (2010), "The Moon Agreement in Current Scenarios. Amendments to Multilateral Treaties. How the Suggestion Amendments be Made", Proceedings of the international Institute of Space Law 2010. 53rd Colloquium on the Law of Outer Space, Praga, República Checa (27 septiembre- 1 octubre), pp. 121-124.

37 Le Gouvernement du Grand-Duché de Luxembourg. Loi du 20 Juillet 2017 sur 1'exploration et utilisation des ressources de 1'espace de Luxembourg, https://egilux.public.lu/eli/etat/leg/loi/2 017/07/20/a674/jo, (consulta:20.04.2021).

38 FROEHLICH, A. y SEFFINGA, V. (Eds.) (2018), National Space Legislation. Studies in Space Policy, Springer International Publishing AG, 15, pp. 128 y 129.

39 Conseil d'État de Luxembourg. Avis 51.987 du 7 avril 2017. Projet de loi sur l'exploration et l'utilisation des ressources de l'espace, p. 8: “(...) Il résulte de ce qui précède que l'exploitation des ressources de l'espace extra-atmosphérique ne bénéficie actuellement pas d'un cadre normatif international qui la réglemente, de sorte qu'on peut douter que le projet de loi sous avis permette d'atteindre l'objectif de la 'sécurité juridique' que les auteurs de la loi en projet entendent établir. Il est vrai que ceci n'a cependant pas empêché les États-Unis d'Amérique de légiférer en la matière, voie que le Luxembourg voudrait également suivre par le biais du projet de loi sous avis", https://conseil-etat.public.lu/fr/avis/2017/04/07042017/51987.html, (consulta: 20.04.2021).

40 La cuestión sobre el estudio de posibles modelos de normas jurídicas para las actividades de exploración, explotación y utilización de los recursos forma parte del programa de la Subcomisión de Asuntos Jurídicos desde el año 2017.

41 UN Doc. A/AC.105/C.2/2021/CRP.22. Propuesta de establecimiento de un grupo de trabajo sobre los recursos espaciales presentada por Alemania, Austria, Bélgica, Chequia, Eslovaquia, España, Grecia, Finlandia, Polonia y Portugal.

42 UN Doc. A/AC.105/C.2/2021/CRP.18. Committee on the Peaceful Uses of Outer Space Legal Subcommittee Sixtieth session Vienna, 31 May-11 June 2021- Item 14 of the provisional agenda- General exchange of views on potential legal models for activities in exploration, exploitation and utilization of space resources The Establishment of a Working Group on Potential Legal Models for Activities in Exploration, Exploitation and Utilization of Space Resources Proposal submitted by China.

43 UN Doc. A/AC.105/C.2/2021/CRP.26. Committee on the Peaceful Uses of Outer Space Legal Subcommittee Sixtieth session Vienna, 31 May-11 June 2021- Item 14 of the provisional agenda- General exchange of views on potential legal models for activities in exploration, 
exploitation and utilization of space resources The Establishment of a Working Group on Potential Legal Models for Activities in Exploration, Exploitation and Utilization of Space Resources. Proposal submitted by Russian Federation.

44 UN Doc. A/AC.105/C.2/L.315. Elementos que podrían constituir una base para la elaboración de un marco internacional sobre las actividades relativas a los recursos espaciales Documento de trabajo presentado por Luxemburgo y los Países Bajos.

45 Audio de las sesiones de la Subcomisión de Asuntos Jurídicos sobre su $58^{\circ}$ período de sesiones, sobre el punto 14 del programa del 5.04.2019 y del 8.04.2019.

46 Universidad de Leiden. The Hague International Space Resources Governance Working Group, https://www.universiteitleiden.nl/en/law/institute-of-public-law/institute-of-air-space-1 aw/the-hague-space-resources-governance-working-group, (consulta:1.06.2021).

47 ESRIC. European Space Resources Innovation Centre. ESRIC is a joint initiative of the Luxembourg Space Agency (LSA) and Luxembourg Institute of Science and Technology (LIST) with the European Space Agency (ESA) as a strategic partner, https://www.esric.lu/, (consulta:1.06.2021).

48 The Hague International Space Resources Governance Working Group; ESRIC. European Space Resources Innovation Centre; Moon Village Association, entre otros.

49 FARAMIÑAN GILBERT, J. M. (2009), "Las actividades en la zona internacional de los fondos marinos y en la luna y otros cuerpos celestes (análisis comparativo del aprovechamiento de los recursos)", en FARAMIÑÁN GILBERT, J. M. y MUÑOZ RODRÍGUEZ, C., Convergencias y divergencias de los regímenes jurídicos del espacio ultraterrestre y de determinados espacios marinos, Universidad de Jaén, Jaén, p. 1-22.

$50 \quad$ PRADO ALEGRE, E. (2018), Los Estados de la Unión Europea frente a los Grandes Retos del Derecho Internacional del Espacio, Instituto Iberoamericano de Derecho Aeronáutico del Espacio y de la Aviación Comercial, Madrid, p. 244.

51 UN Doc. COPUOS/LEGAL/T.803 (22.03.2010), p.13: Intervención Sra. F. MANGIN.

52 MARCHISIO, S. (2010), "The draft code of conduct for outer space activities", United Nations Workshop on Space Law Activities of States in Outer Space in Light of New Developments: Meeting International Responsibilities and Establishing National Legal and Policy Frameworks, 16-19 November, Bangkok, Thailand, https://www.unoosa.org/pdf/pres/2 010/SLW2010/02-10b.pdf, (consulta: 1.05.2021).

53 UN Doc. COPUOS/LEGAL/T.806 (23.03.2010), p. 4: Sr. V. Y. TITUSHKIN (Federación de Rusia): “(...) Quisiera referirme brevemente a una iniciativa, a saber, que se desarrolle un código de conducta. Pensamos que esta idea exige un análisis pormenorizado. Es preciso estudiar el tema, el ámbito de aplicación de este código, los vínculos que existen entre elementos fundamentales y posiciones existentes de derecho espacial internacional. Pensamos que nadie se interesa en un código que se pueda considerar como una alternativa a las disposiciones completas y enteras del derecho espacial. Si este fuera el caso correríamos el riesgo de desfragmentar el derecho espacial y de desestabilizar el régimen existente que rige el uso del espacio ultraterrestre"; UN Doc. COPUOS (LSC) 908th Meeting, script (20.04.2015), p. 17: Mr. GUDONOV (Federación de Rusia): “(...) We support the implementation in national legislation of generally accepted norms of international law but we are against the idea of its stakeholders foisting terms on other countries by stating that if you do not implement this, you are being irresponsible. Therefore, I would like to recall once again the role that is played by the Legal Subcommittee, not just within COPUOS, but within the entire United Nations system.

54 JOHNSON, C. (2014), "Draft International Code of Conduct for Outer Space Activities Fact Sheet". Secure World Foundation, https://swfound.org/media/166384/swf_draft_interna tional_code_of_conduct_for_outer_space_activities_fact_sheet_february_2014.pdf, (consulta: 10.06.2021).

55 PRADO ALEGRE, (2020), p. 15.

56 FARAMIÑÁN GILBERT, (2020), pp. 118 y ss.

57 UN Doc. A/AC.105/C.2/L.314/Add.2 XIII. Intercambio general de opiniones sobre posibles modelos de normas jurídicas sobre las actividades de exploración, explotación y utilización de los recursos espaciales. Comisión sobre la Utilización del Espacio Ultraterrestre con Fines Pacíficos Subcomisión de Asuntos Jurídicos $60^{\circ}$ período de sesiones Viena, 31 de mayo a 11 de junio de 2021. 
ALGUNAS CONSIDERACIONES JURÍDICAS PARA ALCANZAR UN CONSENSO EN TORNO A LA GOBERNANZA DE LA... Elvira PRADO ALEGRE

20

58 WATTLES, J. (12 de julio de 2021), "Virgin Galactic founder Richard Branson successfully rockets to outer space", CNN Business, https:/edition.cnn.com/2021/07/11/tech/richard-brans on-virgin-galactic-space-flight-scn/index.html, (consulta: 12.07.2021).

59 SHEETZ, M. (20 de julio de 2021), "Jeff Bezos reaches space on Blue Origin's first crewed launch”. CNBC, https://www.cnbc.com/2021/07/20/jeff-bezos-reaches-space-on-blue-originsfirst-crewed-launch.html, (consulta: 20.07.2021). 